\title{
Une étude asymptotique probabiliste des coefficients d'une série entière
}

\author{
Bernard Candelpergher, Michel Miniconi \\ Laboratoire J.-A. Dieudonné, UMR 7351 \\ Université Nice Sophia Antipolis, 06108 Nice Cedex 02, France \\ candel@unice.fr, miniconi@unice.fr
}

(15 juillet 2013)

Résumé. En partant des idées de Rosenbloom [7] et Hayman [5], Luis Báez-Duarte donne dans [1] une preuve probabiliste de la formule asymptotique de Hardy-Ramanujan pour les partitions d'un entier. Le principe général de la méthode repose sur la convergence en loi d'une famille de variables aléatoires vers la loi normale. Dans notre travail nous démontrons un théorème de type Liapounov (Chung 2]) qui justifie cette convergence. L'obtention de formules asymptotiques simples nécessite une condition dite Gaussienne forte énoncée par Luis Báez-Duarte, que nous démontrons dans une situation permettant d'obtenir une formule asymptotique classique pour les partitions d'un entier en entiers distincts (Erdös-Lehner [4, Ingham [6]).

Abstract. Following the ideas of Rosenbloom [7] and Hayman [5, Luis Báez-Duarte gives in [1] a probabilistic proof of Hardy-Ramanujan's asymptotic formula for the partitions of an integer. The main principle of the method relies on the convergence in law of a family of random variables to a gaussian variable. In our work we prove a theorem of the Liapounov type (Chung [2]) that justifies this convergence. To obtain simple asymptotic formulæ a condition of the so-called strong Gaussian type defined by Luis Báez-Duarte is required; we demonstrate this in a situation that make it possible to obtain a classical asymptotic formula for the partitions of an integer with distinct parts (Erdös-Lehner [4], Ingham [6]).

\section{Introduction}

\section{Notation.}

On désigne par $\mathcal{O}_{+}(D(0,1))$ l'ensemble des fonctions $f$ analytiques de rayon de convergence 1 telles que $f(t)=\sum_{n \geq 0} a_{n} t^{n}$ avec $a_{n}$ réels positifs non tous nuls. En particulier on a $f(t)>0$ pour tout $\left.t \in\right] 0,1[$.

Définition.

Soit $f \in \mathcal{O}_{+}(D(0,1)):$ pour tout $\left.t \in\right] 0,1[$ on définit la mesure discrète sur $\mathbb{R}$

$$
\mu_{t}(f)=\sum_{n \geq 0} \frac{a_{n} t^{n}}{f(t)} \delta_{n}
$$

On associe à cette mesure une variable aléatoire $X_{t}$ définie sur l'espace probabilisé $\left.\Omega=\right] 0,1[$, à valeurs dans $\mathbb{N}$, telle que

$$
P\left(X_{t}=n\right)=\frac{a_{n} t^{n}}{f(t)}
$$

le but étant de démontrer des résultats sur le comportement asymptotique des $a_{n}$ en utilisant des méthodes probabilistes (voir Rosenbloom [7] qui attribue cette idée à Khinchin).

\section{Moments et fonction caractéristique.}

Les séries $\sum_{n>0} n^{k} a_{n} t^{n}$ étant aussi convergentes dans le disque $D(0,1)$, on en déduit que $X_{t}$ possède un moment d'ordre $k$ pour tout $k \geq 1$. En particulier pour $k=1$ on a, pour tout $t \in] 0,1[$ :

$$
E\left(X_{t}\right)=\sum_{n \geq 0} n \frac{a_{n} t^{n}}{f(t)}=t \frac{f^{\prime}(t)}{f(t)}
$$

On pose $m(t)=E\left(X_{t}\right), 0<t<1$; la fonction $m$ est continue sur $] 0,1[$. 
La fonction caractéristique $\varphi_{X_{t}}\left(\right.$ ou $\left.\varphi_{\mu_{t}(f)}\right)$ de $X_{t}$ est donnée par

$$
\varphi_{X_{t}}(\theta)=E\left(e^{i \theta X_{t}}\right)=\sum_{n \geq 0} e^{i n \theta} \frac{a_{n} t^{n}}{f(t)}=\frac{f\left(t e^{i \theta}\right)}{f(t)}
$$

Les coefficients du développement de Taylor de $f$ sont liés à la fonction caractéristique $\varphi_{X_{t}}$ par

$$
a_{n}=\frac{f(t)}{2 \pi t^{n}} \int_{-\pi}^{\pi} \varphi_{X_{t}}(\theta) e^{-i n \theta} d \theta
$$

pour tout $t \in] 0,1[$. 


\section{Normalisation.}

Soit $\sigma(t)=\sqrt{\operatorname{Var}\left(X_{t}\right)}$ et considérons la variable aléatoire centrée réduite

$$
Z_{t}=\frac{X_{t}-m(t)}{\sigma(t)}
$$

On a

$$
\varphi_{Z_{t}}(x)=e^{-i x \frac{m(t)}{\sigma(t)}} \varphi_{X_{t}}\left(\frac{x}{\sigma(t)}\right)
$$

donc en posant $\theta=\frac{x}{\sigma(t)}$ dans la formule (1.1) donnant $a_{n}$, on obtient pour tout $\left.t \in\right] 0,1[$

$$
a_{n}=\frac{f(t)}{2 \pi \sigma(t) t^{n}} \int_{-\pi \sigma(t)}^{\pi \sigma(t)} \varphi_{Z_{t}}(x) e^{i \frac{x}{\sigma(t)}(m(t)-n)} d x .
$$

Supposons qu'il existe une suite $t_{n} \rightarrow 1$ telle que

$$
m\left(t_{n}\right)=n \text { pour tout } n
$$

alors on a

$$
a_{n}=\frac{f\left(t_{n}\right)}{2 \pi \sigma\left(t_{n}\right)\left(t_{n}\right)^{n}} \int_{-\pi \sigma\left(t_{n}\right)}^{\pi \sigma\left(t_{n}\right)} \varphi_{Z_{t_{n}}}(x) d x
$$

\section{Comportement asymptotique des $a_{n}$ lorsque $n \rightarrow+\infty$}

On suppose que la fonction $m$ est continue, strictement croissante et qu'elle tend vers $+\infty$ lorsque $t$ tend vers 1 . Soit $\left(t_{n}\right)$ une suite dans $] 0,1[$ tendant vers 1 et telle que

$$
m\left(t_{n}\right)=n \quad \text { pour tout } n
$$

et

$$
\sigma\left(t_{n}\right) \rightarrow+\infty
$$

On a alors

$$
a_{n}=\frac{f\left(t_{n}\right)}{2 \pi \sigma\left(t_{n}\right)\left(t_{n}\right)^{n}} \int_{-\pi \sigma\left(t_{n}\right)}^{\pi \sigma\left(t_{n}\right)} \varphi_{Z_{t_{n}}}(x) d x
$$

Supposons en outre que l'on ait la convergence en loi de $Z_{t}$ vers une variable aléatoire $Z$ de loi $\mathrm{N}(0,1)$ quand $t \rightarrow 1$, ce qui veut dire que

$$
\varphi_{Z_{t}}(x) \rightarrow \varphi_{Z}(x)=e^{-\frac{x^{2}}{2}} \text { pour tout } x \in \mathbb{R},
$$

alors on peut espérer un résultat du type (voir Hayman [5])

$$
a_{n} \backsim \frac{f\left(t_{n}\right)}{2 \pi \sigma\left(t_{n}\right)\left(t_{n}\right)^{n}} \int_{-\infty}^{+\infty} e^{-\frac{x^{2}}{2}} d x=\frac{f\left(t_{n}\right)}{\sqrt{2 \pi} \sigma\left(t_{n}\right)\left(t_{n}\right)^{n}}
$$

Pour que la formule ci-dessus donne un équivalent sous une forme analytique simple, il faudrait pouvoir résoudre explicitement l'équation

$$
m\left(t_{n}\right)=n .
$$

Quand ceci n'est pas possible, la stratégie consiste alors à utiliser un équivalent de la fonction $t \mapsto m(t)$ lorsque $t \rightarrow 1$.

Soient $m_{1}$ et $\sigma_{1}$ des équivalents de $m$ et $\sigma$ respectivement lorsque $t \rightarrow 1$ :

$$
\begin{aligned}
m(t) & \sim m_{1}(t) \\
\sigma(t) & \sim \sigma_{1}(t)
\end{aligned}
$$


Soit alors $\left(\tau_{n}\right)$ une suite dans ]0,1[ tendant vers 1 et telle que pour tout $n$

$$
m_{1}\left(\tau_{n}\right)=n
$$

Posons $Z_{t}^{1}=\frac{X_{t}-m_{1}(t)}{\sigma_{1}(t)}$. On a comme précédemment

$$
a_{n}=\frac{f\left(\tau_{n}\right)}{2 \pi \sigma_{1}\left(\tau_{n}\right) \tau_{n}^{n}} \int_{-\sigma_{1}\left(\tau_{n}\right) \pi}^{\sigma_{1}\left(\tau_{n}\right) \pi} E\left(e^{i x Z_{\tau_{n}}^{1}}\right) d x
$$

Sous l'hypothèse

$$
\frac{m\left(\tau_{n}\right)-m_{1}\left(\tau_{n}\right)}{\sigma_{1}\left(\tau_{n}\right)} \rightarrow 0 \text { quand } \tau_{n} \rightarrow 1
$$

et la condition de convergence forte énoncée par Báez-Duarte dans [1] :

$$
\int_{-\sigma\left(\tau_{n}\right) \pi}^{\sigma\left(\tau_{n}\right) \pi}\left|\varphi_{Z_{\tau_{n}}}(x)-e^{-x^{2} / 2}\right| d x \rightarrow 0
$$

on peut alors obtenir

$$
a_{n} \backsim \frac{f\left(\tau_{n}\right)}{\sqrt{2 \pi} \sigma_{1}\left(\tau_{n}\right)\left(\tau_{n}\right)^{n}}
$$

On va appliquer la méthode que l'on vient de décrire à la fonction

$$
f(z)=\sum q(n) z^{n}
$$

où $q(n)$ est le nombre de partitions restreintes de $n$, c'est-à-dire le nombre des décompositions

$$
n=n_{1}+\cdots+n_{p}
$$

en entiers strictement positifs différents les uns des autres afin d'obtenir la formule asymptotique des partitions restreintes :

$$
q(n) \sim \frac{1}{4} \frac{e^{\frac{\pi \sqrt{n}}{\sqrt{3}}}}{3^{1 / 4} n^{3 / 4}}
$$

(voir par exemple Erdös [4] ou Ingham [6]).

\section{Variable associée à un produit infini}

\subsection{Mesure associée à un produit}

Lemme 2.1 Soient $f_{1}$ et $f_{2}$ deux fonctions dans $\mathcal{O}_{+}(D(0,1))$, alors le produit $f_{1} f_{2}$ est dans $\mathcal{O}_{+}(D(0,1))$ et

$$
\mu_{t}\left(f_{1} f_{2}\right)=\mu_{t}\left(f_{1}\right) * \mu_{t}\left(f_{2}\right)
$$

Plus généralement, soient $f_{1}, f_{2}, \ldots$ des fonctions dans $\mathcal{O}_{+}(D(0,1))$, alors le produit $f_{1} \ldots f_{n}$ est $\operatorname{dans} \mathcal{O}_{+}(D(0,1))$ :

$$
\mu_{t}\left(f_{1} \ldots f_{n}\right)=\mu_{t}\left(f_{1}\right) * \cdots * \mu_{t}\left(f_{n}\right)
$$

\section{Démonstration}

Soient $f_{1}(t)=\sum_{n \geq 0} a_{n} t^{n}$ et $f_{2}(t)=\sum_{n \geq 0} b_{n} t^{n}$, on a

$$
f_{1}(t) f_{2}(t)=\sum_{n \geq 0} a_{n} t^{n} \sum_{n \geq 0} b_{n} t^{n}=\sum_{n \geq 0} \sum_{k+l=n} a_{k} b_{l} t^{n}
$$


donc

$$
\mu_{t}\left(f_{1} f_{2}\right)=\sum_{n \geq 0} \frac{\sum_{k+l=n} a_{k} b_{l} t^{n}}{f_{1}(t) f_{2}(t)} \delta_{n}=\sum_{n \geq 0} \sum_{k+l=n} \frac{a_{k} t^{k} b_{l} t^{l}}{f_{1}(t) f_{2}(t)} \delta_{k} * \delta_{l}=\mu_{t}\left(f_{1}\right) * \mu_{t}\left(f_{2}\right)
$$

Par récurrence on a $\mu_{t}\left(f_{1} \ldots f_{n}\right)=\mu_{t}\left(f_{1}\right) * \cdots * \mu_{t}\left(f_{n}\right)$.

Théorème 2.2 Soit $\left(f_{n}\right)$ une suite de fonctions dans $\mathcal{O}_{+}(D(0,1))$ telle que le produit infini $\prod_{k \geq 1}^{+\infty} f_{k}$ converge uniformément sur tout compact de $D(0,1)$. Alors la fonction $f=\prod_{k \geq 1}^{+\infty} f_{k}$ est dans $\mathcal{O}_{+}(D(0,1))$ et la suite des mesures $\mu_{t}\left(f_{1} \ldots f_{n}\right)=\mu_{t}\left(f_{1}\right) * \cdots * \mu_{t}\left(f_{n}\right)$ converge en loi vers la mesure $\mu_{t}(f)$ lorsque $n \rightarrow+\infty$.

Démonstration. Comme le produit infini $\prod_{k \geq 1}^{+\infty} f_{k}$ converge uniformément sur tout compact de $D(0,1)$ on en déduit que la fonction $f=\prod_{k \geq 1}^{+\infty} f_{k}$ est analytique dans $D(0,1)$.

En outre on a

$$
f(z)=\prod_{k \geq 1}^{+\infty} f_{k}(z)=\prod_{k \geq 1}^{+\infty} \sum_{n \geq 0} a_{n, k} z^{n}=\sum_{n \geq 0} z^{n} \sum_{n_{1}+\ldots+n_{p}=n} a_{n_{1}, 1} \ldots a_{n_{p}, p}
$$

donc $f(z)=\sum_{n \geq 0} a_{n} z^{n}$ avec

$$
a_{n}=\sum_{n_{1}+\ldots+n_{p}=n} a_{n_{1}, 1} \ldots a_{n_{p}, p}
$$

ce qui prouve que $f \in \mathcal{O}_{+}(D(0,1))$.

D'autre part, la fonction caractéristique de $\mu_{t}\left(f_{1} \ldots f_{n}\right)=\mu_{t}\left(f_{1}\right) * \cdots * \mu_{t}\left(f_{n}\right)$ est égale au produit des fonctions caractéristiques de chacune des lois

$$
\varphi_{\mu_{t}\left(f_{1}\right) * \cdots * \mu_{t}\left(f_{n}\right)}(x)=\frac{f_{1}\left(t e^{i x}\right)}{f_{1}(t)} \cdots \frac{f_{n}\left(t e^{i x}\right)}{f_{n}(t)}
$$

Comme le produit $f_{1}(z) \ldots f_{n}(z)$ tend vers $f(z)$ pour tout $z$ dans $D(0,1)$, on a pour tout $\left.t \in\right] 0,1[$

$$
\lim _{n \rightarrow+\infty} \frac{f_{1}\left(t e^{i x}\right)}{f_{1}(t)} \cdots \frac{f_{n}\left(t e^{i x}\right)}{f_{n}(t)}=\frac{f\left(t e^{i x}\right)}{f(t)}
$$

La suite des fonctions caractéristiques des mesures $\mu_{t}\left(f_{1} \ldots f_{n}\right)$ converge donc simplement vers la fonction caractéristique de la mesure $\mu_{t}(f)$ associée à $f$.

\subsection{La série des variables aléatoires associées}

Soit $\left(f_{n}\right)$ une suite de fonctions dans $\mathcal{O}_{+}(D(0,1))$ telle que le produit infini $f=\prod_{k \geq 1}^{+\infty} f_{k}$ converge uniformément sur tout compact de $D(0,1)$.

Par un théorème classique, à la suite des mesures de probabilité $\left(\mu_{t}\left(f_{n}\right)\right)$ on peut associer une probabilité sur l'espace produit $\Omega=] 0,1\left[{ }^{\mathbb{N}}\right.$ et une suite de variables aléatoires $\left(X_{n, t}\right)$ à valeurs dans $\mathbb{N}$ indépendantes telle que pour tout $n \geq 1$ la variable aléatoire $X_{n, t}$ ait pour loi $\mu_{t}\left(f_{n}\right)$.

On peut alors affirmer que pour tout $n \geq 1$, la mesure $\mu_{t}\left(f_{1} \ldots f_{n}\right)$ est la loi de la somme $X_{1, t}+\ldots+X_{n, t}$. La convergence de la suite de mesures $\mu_{t}\left(f_{1} \ldots f_{n}\right)$ se traduit donc par la convergence en loi de la série $\sum_{n \geq 1} X_{n, t}$. La loi de $\sum_{n \geq 1} X_{n, t}$ n'est autre que $\mu_{t}(f)$.

D'après le théorème de Kolmogorov, si la série

$$
\sum_{n \geq 1} \sigma^{2}\left(X_{n, t}\right)=\sum_{n \geq 1} \operatorname{Var}\left(X_{n, t}-E\left(X_{n, t}\right)\right)
$$


est convergente, alors la série $\sum_{n>1}\left(X_{n, t}-E\left(X_{n, t}\right)\right)$ converge presque sûrement sur $\Omega$. Si l'on suppose en outre que la série $\sum_{n \geq 1} E\left(X_{n, t}\right)$ converge alors la série $\sum_{n \geq 1} X_{n, t}$ converge presque sûrement.

Les $X_{n, t}$ étant positives et indépendantes, on en déduit par le théorème de Beppo-Levi que

$$
\operatorname{Var}\left(\sum_{n \geq 1} X_{n, t}\right)=\sum_{n \geq 1} \operatorname{Var}\left(X_{n, t}\right)
$$

Sous ces hypothèses on peut alors donner la définition suivante :

Définition.

Soit $X_{t}$ la variable aléatoire définie comme la somme de la série $\sum_{n \geq 1} X_{n, t}$ dont la loi sera notée $\mu_{t}(f)$. On pose

$$
m(t)=E\left(X_{t}\right)=\sum_{n \geq 1} E\left(X_{n, t}\right) \quad \text { et } \quad \sigma(t)=\left(\operatorname{Var}\left(X_{t}\right)\right)^{1 / 2}=\left(\sum_{n \geq 1} \operatorname{Var}\left(X_{n, t}\right)\right)^{1 / 2}
$$

\subsection{Application à l'étude des coefficients}

On se donne une suite de fonctions $f_{n}=\sum_{n \geq 0} a_{k, n} z^{k}$ dans $\mathcal{O}_{+}(D(0,1))$ telle que le produit infini $\prod_{n \geq 1}^{+\infty} f_{n}$ converge uniformément sur tout compact de $D(0,1)$. La fonction $f=\prod_{n \geq 1}^{+\infty} f_{n}$ est dans $\mathcal{O}_{+}(D(0,1))$ et l'on peut écrire $f(z)=\sum_{n \geq 0} a_{n} z^{n}$ avec

$$
a_{n}=\sum_{n_{1}+\ldots+n_{p}=n} a_{n_{1}, 1} \ldots a_{n_{p}, p}
$$

Si les séries $\sum_{n \geq 1} E\left(X_{n, t}\right)$ et $\sum_{n \geq 1} \sigma^{2}\left(X_{n, t}\right)$ sont convergentes, considérons la variable aléatoire centrée réduite

$$
Z_{t}=\frac{\sum_{n \geq 1} X_{n, t}-m(t)}{\sigma(t)}
$$

Supposons que $Z_{t}$ converge en loi quand $t \rightarrow 1$ vers une variable aléatoire $Z$ de loi N $(0,1)$ et soit $\left(t_{n}\right)$ une suite tendant vers 1 telle que

$$
m\left(t_{n}\right)=n \text { pour tout } n .
$$

On a alors

$$
\sum_{n_{1}+\ldots+n_{p}=n} a_{n_{1}, 1 \ldots} a_{n_{p}, p}=\frac{f\left(t_{n}\right)}{2 \pi \sigma\left(t_{n}\right)\left(t_{n}\right)^{n}} \int_{-\pi \sigma\left(t_{n}\right)}^{\pi \sigma\left(t_{n}\right)} \varphi_{Z_{t_{n}}}(x) d x
$$

Si $\sigma\left(t_{n}\right) \rightarrow+\infty$ quand $t_{n} \rightarrow 1$ il est plausible que

$$
\lim _{t_{n} \rightarrow 1} \int_{-\sigma\left(t_{n}\right) \pi}^{\sigma\left(t_{n}\right) \pi} \varphi_{t_{n}}(x) d x=\int_{-\infty}^{+\infty} \lim \varphi_{t_{n}}(x) d x=\int_{-\infty}^{+\infty} e^{-x^{2} / 2} d x=\sqrt{2 \pi}
$$

et on en déduirait ainsi la formule asymptotique des coefficients

$$
\sum_{n_{1}+\ldots+n_{p}=n} a_{n_{1}, 1 \ldots} a_{n_{p}, p} \sim \frac{f\left(t_{n}\right)}{\sqrt{2 \pi} \sigma\left(t_{n}\right)\left(t_{n}\right)^{n}} .
$$

Passage par des équivalents.

Nous détaillons ici la méthode de Luis Báez-Duarte [1]. 
Afin d'établir la formule (2.1) ci-dessus avec $\sigma_{1}$ à la place de $\sigma$ (et $\tau_{n}$ à la place de $t_{n}$ ), il reste à montrer que

$$
\lim _{n \rightarrow+\infty} \int_{-\sigma_{1}\left(\tau_{n}\right) \pi}^{\sigma_{1}\left(\tau_{n}\right) \pi} E\left(e^{i x Z_{\tau_{n}}^{1}}\right) d x=\sqrt{2 \pi}
$$

On remarque que l'on peut écrire

$$
Z_{t}^{1}=\frac{X_{t}-m_{1}(t)}{\sigma_{1}(t)}=Z_{t} \frac{\sigma(t)}{\sigma_{1}(t)}+\varepsilon(t)
$$

où $\varepsilon(t)=\frac{m(t)-m_{1}(t)}{\sigma_{1}(t)}$. On a alors

$$
\begin{aligned}
\int_{-\sigma_{1}\left(\tau_{n}\right) \pi}^{\sigma_{1}\left(\tau_{n}\right) \pi} E\left(e^{\left.i x Z_{\tau_{n}}^{1}\right) d x}\right. & =\int_{-\sigma_{1}\left(\tau_{n}\right) \pi}^{\sigma_{1}\left(\tau_{n}\right) \pi} E\left(e^{i x \frac{\sigma\left(\tau_{n}\right)}{\sigma_{1}\left(\tau_{n}\right)} Z_{\tau_{n}}}\right) e^{i x \varepsilon\left(\tau_{n}\right)} d x \\
& =\frac{\sigma_{1}\left(\tau_{n}\right)}{\sigma\left(\tau_{n}\right)} \int_{-\sigma\left(\tau_{n}\right) \pi}^{\sigma\left(\tau_{n}\right) \pi} \varphi_{Z_{\tau_{n}}}(x) e^{i x \frac{\sigma_{1}\left(\tau_{n}\right)}{\sigma\left(\tau_{n}\right)} \varepsilon\left(\tau_{n}\right)} d x
\end{aligned}
$$

Pour justifier le remplacement par des équivalents on énonce deux hypothèses :

Hypothèse 1. Supposons que

$$
\frac{m\left(\tau_{n}\right)-m_{1}\left(\tau_{n}\right)}{\sigma_{1}\left(\tau_{n}\right)}=\varepsilon\left(\tau_{n}\right) \rightarrow 0 \text { quand } \tau_{n} \rightarrow 1
$$

Hypothèse 2. Supposons que

$$
\int_{-\sigma\left(\tau_{n}\right) \pi}^{\sigma\left(\tau_{n}\right) \pi}\left|\varphi_{Z_{\tau_{n}}}(x)-e^{-x^{2} / 2}\right| d x \rightarrow 0
$$

Sous ces deux hypothèses il est facile de montrer que la suite $\int_{-\sigma\left(\tau_{n}\right) \pi}^{\sigma\left(\tau_{n}\right) \pi} \varphi_{Z_{\tau_{n}}}(x) e^{i x \frac{\sigma_{1}\left(\tau_{n}\right)}{\sigma\left(\tau_{n}\right)} \varepsilon\left(\tau_{n}\right)} d x$ converge vers $\sqrt{2 \pi}$.

En effet, on a

$$
\begin{aligned}
& \left|\int_{-\sigma\left(\tau_{n}\right) \pi}^{\sigma\left(\tau_{n}\right) \pi} \varphi_{Z_{\tau_{n}}}(x) e^{i x \frac{\sigma_{1}\left(\tau_{n}\right)}{\sigma\left(\tau_{n}\right)} \varepsilon\left(\tau_{n}\right)} d x-\int_{-\sigma\left(\tau_{n}\right) \pi}^{\sigma\left(\tau_{n}\right) \pi} e^{i x \frac{\sigma_{1}\left(\tau_{n}\right)}{\sigma\left(\tau_{n}\right)} \varepsilon\left(\tau_{n}\right)} e^{-x^{2} / 2} d x\right| \\
\leq & \int_{-\sigma\left(\tau_{n}\right) \pi}^{\sigma\left(\tau_{n}\right) \pi}\left|\varphi_{Z_{\tau_{n}}}(x)-e^{-x^{2} / 2}\right| d x
\end{aligned}
$$

il suffit donc de montrer que

$$
\lim _{\tau_{n} \rightarrow 1} \int_{-\sigma\left(\tau_{n}\right) \pi}^{\sigma\left(\tau_{n}\right) \pi} e^{i x \frac{\sigma_{1}\left(\tau_{n}\right)}{\sigma\left(\tau_{n}\right)} \varepsilon\left(\tau_{n}\right)} e^{-x^{2} / 2} d x=\sqrt{2 \pi} .
$$

Ceci résulte du théorème de la convergence dominée, car on a

$$
\lim _{\tau_{n} \rightarrow 1} e^{i x \frac{\sigma_{1}\left(\tau_{n}\right)}{\sigma\left(\tau_{n}\right)} \varepsilon\left(\tau_{n}\right)} e^{-x^{2} / 2}=e^{-x^{2} / 2}
$$

et

$$
\left|e^{i x \frac{\sigma_{1}\left(\tau_{n}\right)}{\sigma\left(\tau_{n}\right)} \varepsilon\left(\tau_{n}\right)} e^{-x^{2} / 2}\right| \leq e^{-x^{2} / 2}
$$

Résumons ce qui précède dans le théorème suivant : 
Théorème 2.3 (Théorème des équivalents) Soit $\left(f_{n}=\sum_{n \geq 0} a_{k, n} z^{k}\right)$ une suite de fonctions dans $\mathcal{O}_{+}(D(0,1))$ telle que le produit infini $\prod_{n \geq 1}^{+\infty} f_{n}$ converge uniformément sur tout compact de $D(0,1)$. La fonction $f=\prod_{n \geq 1}^{+\infty} f_{n}$ est donc analytique dans $D(0,1)$ et $f(z)=\sum_{n \geq 0} a_{n} z^{n}$ avec

$$
a_{n}=\sum_{n_{1}+\ldots+n_{p}=n} a_{n_{1}, 1} \ldots a_{n_{p}, p}
$$

Si les séries $\sum_{n \geq 1} E\left(X_{n, t}\right)$ et $\sum_{n \geq 1} \sigma^{2}\left(X_{n, t}\right)$ sont convergentes, considérons la variable aléatoire centrée réduite

$$
Z_{t}=\frac{\sum_{n \geq 1} X_{n, t}-m(t)}{\sigma(t)}
$$

Supposons que $Z_{t}$ converge en loi quand $t \rightarrow 1$ vers une variable aléatoire $Z$ de loi $\mathbf{N}(\mathbf{0}, \mathbf{1})$ avec la condition de convergence forte (voir [1]) :

$$
\lim _{t \rightarrow 1} \int_{-\sigma(t) \pi}^{\sigma(t) \pi}\left|\varphi_{Z_{t}}(x)-e^{-x^{2} / 2}\right| d x=0
$$

Soient $m_{1}$ et $\sigma_{1}$ des équivalents de $m$ et $\sigma$ respectivement lorsque $t \rightarrow 1$ :

$$
\begin{aligned}
m(t) & \sim m_{1}(t) \\
\sigma(t) & \sim \sigma_{1}(t)
\end{aligned}
$$

Soit une suite $\left(\tau_{n}\right)$ dans $] 0,1[$ convergeant vers 1 et telle que pour tout $n$ on ait :

$$
m_{1}\left(\tau_{n}\right)=n
$$

avec

$$
\frac{m\left(\tau_{n}\right)-m_{1}\left(\tau_{n}\right)}{\sigma_{1}\left(\tau_{n}\right)} \rightarrow 0 \text { quand } \tau_{n} \rightarrow 1
$$

Alors

$$
a_{n} \sim \frac{f\left(\tau_{n}\right)}{\sqrt{2 \pi} \sigma_{1}\left(\tau_{n}\right) \tau_{n}^{n}}
$$

\section{Un théorème de convergence}

Nous énonçons et démontrons un théorème du type Liapounov (voir Chung [2] p. 205 sq.) de convergence vers une loi normale concernant une famille continue de suites infinies de variables aléatoires.

Théorème 3.1 (Théorème de convergence) Soit une suite de variables aléatoires positives $\left(X_{n, t}\right)_{n}$ dans $L^{3}(\Omega)$ telle que pour tout $\left.t \in\right] 0,1[$ :

a) les $X_{n, t}$ sont indépendantes

b) les séries $m(t)=\sum_{n \geq 1} E\left(X_{n, t}\right), \sigma^{2}(t)=\sum_{n \geq 1} \operatorname{Var}\left(X_{n, t}\right)$ et $\Gamma_{3}(t)=\sum_{n \geq 1} E\left(\left|X_{n, t}-E\left(X_{n, t}\right)\right|^{3}\right)$ sont convergentes

c) la fonction $t \mapsto \frac{\Gamma_{3}(t)}{(\sigma(t))^{3}}$ tend vers 0 quand $t \rightarrow 1$

d) $\lim _{t \rightarrow 1} \sup _{n \geq 1} \frac{\operatorname{Var}\left(X_{n, t}\right)}{\sigma^{2}(t)}=0$ 
Alors la série $X_{t}=\sum_{n>1} X_{n, t}$ est convergente presque sûrement et la fonction caractéristique $\varphi_{Z_{t}}$ de la variable aléatoire

$$
Z_{t}=\frac{X_{t}-m(t)}{\sigma(t)}
$$

est telle que

$$
\varphi_{Z_{t}}(x) \rightarrow e^{-x^{2} / 2} \quad \text { quand } \quad t \rightarrow 1
$$

\section{Démonstration.}

Posons $Y_{n, t}=X_{n, t}-E\left(X_{n, t}\right)$ on a $Z_{t}=\frac{\sum_{n \geq 1} Y_{n, t}}{\sigma(t)}$.

Par l'indépendance des $Y_{n, t}$ on voit que la variable aléatoire $Z_{t}$ a pour fonction caractéristique

$$
\varphi_{Z_{t}}(\theta)=E\left(e^{i \theta \sum \frac{Y_{n, t}}{\sigma(t)}}\right)=\prod_{n \geq 1} E\left(e^{i \theta \frac{Y_{n, t}}{\sigma(t)}}\right)
$$

On a $E\left(Y_{n, t}\right)=0$ et $E\left(Y_{n, t}^{2}\right)=\sigma_{n, t}^{2}=\operatorname{Var}\left(X_{n, t}\right)$.

Lemme 3.2 Sous les hypothèses du théorème (3.1) ci-dessus, on a

$$
E\left(e^{i \theta \frac{Y_{n, t}}{\sigma(t)}}\right)=1-\frac{\theta^{2}}{2}\left(\frac{\sigma_{n, t}}{\sigma(t)}\right)^{2}+L_{n}(\theta, t)
$$

avec

$$
\left|L_{n}(\theta, t)\right| \leq \frac{|\theta|^{3}}{6(\sigma(t))^{3}} E\left(\left|Y_{n, t}\right|^{3}\right)
$$

Ce lemme résulte de la formule de Taylor

$$
e^{i x}=1+i x-\frac{x^{2}}{2}-i \int_{0}^{1} \frac{(1-u)^{2}}{2} x^{3} e^{i u x} d u
$$

qui nous donne

$$
e^{i \theta \frac{Y_{n, t}}{\sigma(t)}}=1+i \theta \frac{Y_{n, t}}{\sigma(t)}-\frac{\theta^{2}\left(\frac{Y_{n, t}}{\sigma(t)}\right)^{2}}{2}-i \int_{0}^{1} \frac{(1-u)^{2}}{2} \theta^{3}\left(\frac{Y_{n, t}}{\sigma(t)}\right)^{3} e^{i u \theta \frac{Y_{n, t}}{\sigma(t)}} d u
$$

Comme $E\left(Y_{n, t}\right)=0$ on en déduit que

$$
\begin{aligned}
E\left(e^{i \theta \frac{Y_{n, t}}{\sigma(t)}}\right) & =1-\frac{\theta^{2}\left(\frac{\sigma_{n, t}}{\sigma(t)}\right)^{2}}{2}-i \int_{0}^{1} \frac{(1-u)^{2}}{2} \theta^{3} E\left(\left(\frac{Y_{n, t}}{\sigma(t)}\right)^{3} e^{i u \theta \frac{Y_{n, t}}{\sigma(t)}}\right) d u \\
& =1-\frac{\theta^{2}}{2}\left(\frac{\sigma_{n, t}}{\sigma(t)}\right)^{2}+L_{n}(\theta, t)
\end{aligned}
$$

où

$$
L_{n}(\theta, t)=-i \int_{0}^{1} \frac{(1-u)^{2}}{2} \theta^{3} E\left(\left(\frac{Y_{n, t}}{\sigma(t)}\right)^{3} e^{i u \theta \frac{Y_{n, t}}{\sigma(t)}}\right) d u
$$

On a ainsi la majoration

$$
\left|L_{n}(\theta, t)\right| \leq\left|\theta^{3}\right| E\left(\left(\frac{\left|Y_{n, t}\right|}{\sigma(t)}\right)^{3}\right) \int_{0}^{1} \frac{(1-u)^{2}}{2} d u \leq \frac{|\theta|^{3}}{6(\sigma(t))^{3}} E\left(\left|Y_{n, t}\right|^{3}\right)
$$

Ce qui termine la démonstration du lemme. 
Par l'indépendance des $Y_{n, t}$ la fonction caractéristique $\varphi_{Z_{t}}$ de la variable aléatoire $Z_{t}$ peut s'écrire

$$
\varphi_{Z_{t}}(\theta)=\prod_{n \geq 1}\left(1-\frac{\theta^{2}}{2}\left(\frac{\sigma_{n, t}}{\sigma(t)}\right)^{2}+L_{n}(\theta, t)\right)
$$

Pour montrer que $\varphi_{Z_{t}}(\theta) \rightarrow e^{-\frac{\theta^{2}}{2}}$ quand $t \rightarrow 1$ nous allons utiliser le lemme suivant (dont nous donnons la démonstration dans l'Appendice (voir section 5)) :

Lemme 3.3 Soit $\left(u_{n, t}\right)_{n \geq 1}$ une famille de suites complexes indexées par $\left.t \in\right] 0,1[$ telle que ( $) \sup _{n \geq 1}\left|u_{n, t}\right| \rightarrow 0$ quand $t \rightarrow 1$.

(ı) il existe $M>0$ et $0<\alpha<1$ tel que $\sum_{n \geq 1}\left|u_{n, t}\right| \leq M$ pour tout $\left.t \in\right] \alpha, 1[$.

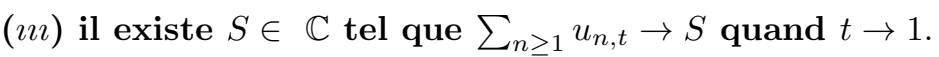

Alors

$$
\lim _{t \rightarrow 1} \prod_{n \geq 1}\left(1+u_{n, t}\right)=e^{S}
$$

On va appliquer ce lemme à la fonction caractéristique (3.1) en posant

$$
u_{n, t}(\theta)=-\frac{\theta^{2}}{2}\left(\frac{\sigma_{n, t}}{\sigma(t)}\right)^{2}+L_{n}(\theta, t)
$$

Vérifions les trois conditions du lemme :

( $)$ On a

$$
\begin{aligned}
\sup _{n \geq 1}\left|u_{n, t}(\theta)\right| & \leq \frac{\theta^{2}}{2} \sup _{n \geq 1}\left(\frac{\sigma_{n, t}}{\sigma(t)}\right)^{2}+\sup _{n \geq 1} L_{n}(\theta, t) \\
& \leq \frac{\theta^{2}}{2} \sup _{n \geq 1}\left(\frac{\sigma_{n, t}}{\sigma(t)}\right)^{2}+\frac{|\theta|^{3}}{6(\sigma(t))^{3}} \sum_{n \geq 1} E\left(\left|Y_{n, t}\right|^{3}\right)
\end{aligned}
$$

D'après les hypothèses $\mathrm{c}$ ) et $\mathrm{d}$ ) du théorème cette dernière quantité tend vers 0 quand $t \rightarrow 1$.

(ıi) On a

$$
\begin{aligned}
\sum_{n \geq 1}\left|u_{n, t}(\theta)\right| & \leq \frac{\theta^{2}}{2} \sum_{n \geq 1}\left(\frac{\sigma_{n, t}}{\sigma(t)}\right)^{2}+\sum_{n \geq 1} L_{n}(\theta, t) \\
& \leq \frac{\theta^{2}}{2}+\frac{|\theta|^{3}}{6(\sigma(t))^{3}} \sum_{n \geq 1} E\left(\left|Y_{n, t}\right|^{3}\right)
\end{aligned}
$$

Or d'après c) la quantité $\frac{1}{(\sigma(t))^{3}} \sum_{n \geq 1} E\left(\left|Y_{n, t}\right|^{3}\right)$ est bornée au voisinage de 1 .

(ıı) On a

$$
\sum_{n \geq 1} u_{n, t}(\theta)=-\frac{\theta^{2}}{2} \sum_{n \geq 1}\left(\frac{\sigma_{n, t}}{\sigma(t)}\right)^{2}+\sum_{n \geq 1} L_{n}(\theta, t)=\frac{\theta^{2}}{2}+\sum_{n \geq 1} L_{n}(\theta, t)
$$

et $\sum_{n \geq 1} L_{n}(\theta, t) \rightarrow 0$ quand $t \rightarrow 1$ par c).

On a donc $\sum_{n \geq 1} u_{n, t}(\theta) \rightarrow-\frac{\theta^{2}}{2}$ quand $t \rightarrow 1$ et par le lemme (3.3)

$$
\varphi_{Z_{t}}(\theta)=\prod_{n \geq 1}\left(1-\frac{\theta^{2}}{2}\left(\frac{\sigma_{n, t}}{\sigma(t)}\right)^{2}+L_{n}(\theta, t)\right) \rightarrow e^{-\frac{\theta^{2}}{2}}
$$




\section{Application aux partitions restreintes}

Considérons la fonction définie par le produit infini

$$
f(z)=\prod_{n \geq 1}^{+\infty}\left(1+z^{n}\right)
$$

Cette fonction est analytique dans $D(0,1)$ car la série $\sum_{n=1}^{+\infty} z^{n}$ converge uniformément sur tout compact de $D(0,1)$. On a

$$
f(z)=\sum q(n) z^{n}
$$

où $q(n)$ est le nombre de partitions restreintes de $n$, c'est-à-dire le nombre des décompositions $n=$ $n_{1}+\cdots+n_{p}$ en entiers strictement positifs différents les uns des autres.

Le but de ce qui suit est d'appliquer la méthode décrite au début de cet article pour obtenir la formule asymptotique des partitions restreintes :

$$
q(n) \sim \frac{1}{4} \frac{e^{\frac{\pi \sqrt{n}}{\sqrt{3}}}}{3^{1 / 4} n^{3 / 4}}
$$

Soit la mesure de probabilité associée à $f_{n}(t)=1+t^{n}$

$$
\mu_{t}\left(f_{n}\right)=\frac{1}{1+t^{n}} \delta_{0}+\frac{t^{n}}{1+t^{n}} \delta_{n}
$$

où $\delta_{0}$ et $\delta_{n}$ représentent les mesures de Dirac en 0 et $n$ respectivement.

On associe à ces mesures une suite de variables aléatoires indépendantes $\left(X_{n, t}\right)$ (voir section (2.2) ). La variable $X_{n, t}$ prend les valeurs 0 et $n$ et on a

$$
\begin{aligned}
E\left(X_{n, t}\right) & =\frac{n t^{n}}{1+t^{n}} \\
\operatorname{Var}\left(X_{n, t}\right) & =\frac{n^{2} t^{n}}{\left(1+t^{n}\right)^{2}}
\end{aligned}
$$

Dans ce qui suit on posera

$$
t=e^{-r}
$$

où $r>0$, de sorte que l'on a

$$
t \rightarrow 1 \Leftrightarrow r \rightarrow 0
$$

\subsection{Vérification des hypothèses du théorème de convergence}

Les séries

$$
m(t)=\sum_{n \geq 1} E\left(X_{n, t}\right)=\sum_{n=1}^{+\infty} \frac{n t^{n}}{1+t^{n}} \quad \text { et } \quad \sigma^{2}(t)=\sum_{n \geq 1} \sigma^{2}\left(X_{n, t}\right)=\sum_{k \geq 1} \frac{n^{2} t^{n}}{\left(1+t^{n}\right)^{2}}
$$

sont clairement convergentes.

Examinons la série $\sum_{n \geq 1} E\left(\left|X_{n, t}-E\left(X_{n, t}\right)\right|^{3}\right)$ : on a

$$
\begin{aligned}
E\left(\left|X_{n, t}-E\left(X_{n, t}\right)\right|^{3}\right) & =\left(\frac{n t^{n}}{1+t^{n}}\right)^{3} \frac{1}{1+t^{n}}+\left(n-\frac{n t^{n}}{1+t^{n}}\right)^{3} \frac{t^{n}}{1+t^{n}} \\
& =n^{3} \frac{t^{3 n}+t^{n}}{\left(1+t^{n}\right)^{4}}
\end{aligned}
$$

donc la série $\sum_{n \geq 1} E\left(\left|X_{n, t}-E\left(X_{n, t}\right)\right|^{3}\right)$ est convergente.

Ainsi les hypothèses a) et b) du théorème de convergence (3.1) sont bien vérifiées. 


\subsubsection{Comportement asymptotique de $m$ et $\sigma^{2}$}

Pour déterminer le comportement asymptotique quand $t \rightarrow 1$ des fonctions $m(t)$ et $\sigma^{2}(t)$ on va utiliser la formule d'Euler-McLaurin rappelée ci-dessous :

si $f \in C^{1}[0,+\infty[$ on a pour tout entier $n \geq 1$

$$
\sum_{k=1}^{n} f(k)=\int_{1}^{n} f(x) d x+\frac{1}{2}(f(1)+f(n))+\int_{1}^{n} b_{1}(x) f^{\prime}(x) d x
$$

où $b_{1}(x)=x-[x]-\frac{1}{2}$. Si en outre $\sum_{k=1}^{+\infty} f(k)$ et $\int_{1}^{+\infty} f(x) d x$ sont convergentes alors

$$
\sum_{k=1}^{+\infty} f(k)=\int_{0}^{+\infty} f(x) d x+\int_{1}^{+\infty} b_{1}(x) f^{\prime}(x) d x+C
$$

où $C=\frac{1}{2} f(1)-\int_{0}^{1} f(x) d x$.

Les fonctions $f$ auxquelles on va appliquer cette formule seront du type

$$
f(x)=\frac{x^{p} e^{-a r x}}{\left(1+e^{-r x}\right)^{q}}
$$

où $a, p, q$ sont des entiers supérieurs ou égaux à 1 . Comme $f(x)=\frac{1}{r^{p}} g(r x)$ où $g(u)=\frac{u^{p} e^{-a u}}{\left(1+e^{-u}\right)^{q}}$, on a

$$
\int_{0}^{+\infty} f(x) d x=\frac{1}{r^{p+1}} \int_{0}^{+\infty} g(u) d u
$$

et

car la fonction $g^{\prime}$ est intégrable.

$$
\left|\int_{1}^{+\infty} b_{1}(x) f^{\prime}(x) d x\right|=\frac{1}{r^{p}}\left|\int_{1}^{+\infty} b_{1}\left(\frac{u}{r}\right) g^{\prime}(u) d u\right| \leq \frac{1}{2 r^{p}} \int_{1}^{+\infty}\left|g^{\prime}(u)\right| d u
$$

La formule d'Euler-MacLaurin nous donne pour $r \rightarrow 0+$

$$
\sum_{k=1}^{+\infty} \frac{k^{p} e^{-a k r}}{\left(1+e^{-k r}\right)^{q}}=\frac{1}{r^{p+1}} \int_{0}^{+\infty} \frac{u^{p} e^{-a u}}{\left(1+e^{-u}\right)^{q}}+O\left(\frac{1}{r^{p}}\right) .
$$

Pour $a=p=q=1$ on obtient

$$
m\left(e^{-r}\right)=\sum_{k=1}^{+\infty} \frac{k e^{-r k}}{1+e^{-r k}}=\frac{1}{r^{2}} \int_{0}^{+\infty} \frac{u e^{-u}}{1+e^{u}} d x+O\left(\frac{1}{r}\right)
$$

Notons que

$$
\frac{1}{r^{2}} \int_{0}^{+\infty} \frac{u e^{-u}}{1+e^{u}} d x=\frac{1}{r^{2}} \sum_{n=0}^{+\infty}(-1)^{n} \int_{0}^{+\infty} u e^{-u(n+1)} d x=\frac{1}{r^{2}} \sum_{n=0}^{+\infty}(-1)^{n} \frac{1}{(n+1)^{2}}=\frac{1}{r^{2}} \frac{\pi^{2}}{12}
$$

On a ainsi

$$
m\left(e^{-r}\right)=m_{1}\left(e^{-r}\right)+O\left(\frac{1}{r}\right) \quad \text { avec } \quad m_{1}\left(e^{-r}\right)=\frac{\pi^{2}}{12} \frac{1}{r^{2}}
$$

Et de la même manière, on a

car

$$
\sigma^{2}\left(e^{-r}\right) \sim \int_{1}^{+\infty} \frac{x^{2} e^{-r x}}{\left(1+e^{-r x}\right)^{2}} d x \sim_{r \rightarrow 0} \frac{\pi^{2}}{6} \frac{1}{r^{3}}=\sigma_{1}^{2}\left(e^{-r}\right)
$$

$$
\int_{0}^{+\infty} \frac{x^{2} e^{-r x}}{\left(1+e^{-r x}\right)^{2}} d x=\sum_{n=1}^{+\infty}(-1)^{n-1} n \int_{0}^{+\infty} x^{2} e^{-r x n} d x=\frac{2}{r^{3}} \sum_{n=1}^{+\infty}(-1)^{n-1} \frac{1}{n^{2}}
$$




\subsubsection{Les conditions c) et $\mathrm{d}$ )}

Calculons $\Gamma_{3}(t)=\sum_{n \geq 1} E\left(\left|X_{n, t}-E\left(X_{n, t}\right)\right|^{3}\right)$ :

$$
\sum_{n \geq 1} E\left(\left|X_{n, t}-E\left(X_{n, t}\right)\right|^{3}\right)=\sum_{n \geq 1} n^{3} \frac{e^{-3 n r}+e^{-n r}}{\left(1+e^{-n r}\right)^{4}} \sim \int_{0}^{+\infty} \frac{x^{3}\left(e^{-3 r x}+e^{-r x}\right)}{\left(1+e^{-r x}\right)^{4}} d x=\frac{C}{r^{4}}
$$

et donc

$$
\frac{\Gamma_{3}(t)}{\sigma(t)^{3}} \sim \frac{\frac{C}{r^{4}}}{\left(\frac{\pi^{2}}{6} \frac{1}{r^{3}}\right)^{3 / 2}}=C_{3} r^{1 / 2}
$$

On a donc bien $\frac{\Gamma_{3}(t)}{\sigma(t)^{3}} \rightarrow 0$ quand $t \rightarrow 1$.

Il reste à voir que $\lim _{t \rightarrow 1} \sup _{n \geq 1} \frac{\operatorname{Var}\left(X_{n, t}\right)}{\sigma^{2}(t)}=0$. On a

$$
\frac{\operatorname{Var}\left(X_{n, t}\right)}{\sigma^{2}(t)}=\frac{1}{\sigma^{2}(t)} n^{2} \frac{t^{n}}{\left(1+t^{n}\right)^{2}} \leq \frac{1}{\sigma^{2}(t)} n^{2} t^{n}
$$

Or on a $n^{2} e^{-n r} \leq \frac{4}{r^{2}} e^{-2}$ pour tout $n$ et $\sigma^{2}\left(e^{-r}\right) \sim \frac{\pi^{2}}{6} \frac{1}{r^{3}}$ d'après (4.1) donc

$$
\limsup _{t \rightarrow 1} \frac{\operatorname{Var}\left(X_{n, t}\right)}{\sigma^{2}(t)}=0
$$

Ainsi les hypothèses du théorème de convergence (3.1) sont bien vérifiées. Par conséquent la fonction caractéristique $\varphi_{Z_{t}}$ de la variable aléatoire

$$
Z_{t}=\frac{\sum_{n \geq 1} X_{n, t}-m(t)}{\sigma(t)}
$$

converge vers $e^{-x^{2} / 2}$ quand $t \rightarrow 1$.

\subsection{Vérification de la condition de convergence forte}

Pour obtenir une formule asymptotique du nombre de partitions restreintes $q(n)$ défini au début de ce paragraphe (4), on doit vérifier les hypothèses du théorème des équivalents, en particulier la condition de convergence forte :

$$
\lim _{t \rightarrow 1} \int_{-\pi \sigma(t)}^{\pi \sigma(t)}\left|\varphi_{Z_{t}}(\theta)-e^{-\theta^{2} / 2}\right| d \theta=0
$$

Pour cela on va décomposer l'intégrale précédente en

$$
\int_{|\theta| \leq \frac{C}{r^{1 / 2}}}\left|\varphi_{Z_{t}}(\theta)-e^{-\theta^{2} / 2}\right| d \theta+\int_{\frac{C}{r^{1 / 2} \leq|\theta| \leq \pi \sigma(t)}}\left|\varphi_{Z_{t}}(\theta)-e^{-\theta^{2} / 2}\right| d \theta
$$

et majorer $\left|\varphi_{Z_{t}}(\theta)\right|$ sur chacun des domaines d'intégration.

Lemme 4.1 Si $|\theta| \leq \frac{1}{4 \frac{\Gamma_{3}(t)}{\sigma^{3}(t)}} \sim \frac{1}{4 C_{3}} r^{-1 / 2}$ alors $\left|\varphi_{Z_{t}}(\theta)\right| \leq e^{-\theta^{2} / 3}$.

Démonstration.

Posons $Y_{n, t}=X_{n, t}-E\left(X_{n, t}\right)$ on a $Z_{t}=\frac{\sum_{n>1} Y_{n, t}}{\sigma(t)}$. On a

$$
\varphi_{Z_{t}}(\theta)=\prod_{n \geq 1} \varphi_{Y_{n, t}}\left(\frac{\theta}{\sigma(t)}\right)
$$

Pour majorer $\left|\varphi_{Z_{t}}(\theta)\right|$ on va utiliser le lemme suivant dont la démonstration est reportée à la section (5.2) : 
Lemme 4.2 (Lemme de Cramér) Soit $Z$ une variable aléatoire centrée telle que $E\left(|Z|^{3}\right)<+\infty$ et $\varphi_{Z}$ sa fonction caractéristique. On a

$$
\left|\varphi_{Z}(\xi)\right|^{2} \leq e^{-\xi^{2} E\left(Z^{2}\right)+\frac{4}{3}|\xi|^{3} E\left(|Z|^{3}\right)}
$$

En particulier si $|\xi| \leq \frac{1}{2} \frac{E\left(Z^{2}\right)}{E\left(|Z|^{3}\right)}$ alors $\left|\varphi_{Z}(\xi)\right|^{2} \leq e^{-\frac{\xi^{2}}{3} E\left(Z^{2}\right)}$.

En appliquant ce lemme on obtient ainsi

$$
\left|\varphi_{Y_{n, t}}\left(\frac{\theta}{\sigma(t)}\right)\right|^{2} \leq \exp \left(-\frac{\sigma_{n, t}^{2}}{\sigma^{2}(t)} \theta^{2}+\frac{4}{3} \frac{|\theta|^{3} E\left(\left|Y_{n, t}\right|^{3}\right)}{\sigma^{3}(t)}\right)
$$

donc

$$
\left|\varphi_{Z_{t}}(\theta)\right|^{2} \leq \prod_{n \geq 1} \exp \left(-\frac{\sigma_{n, t}^{2}}{\sigma^{2}(t)} \theta^{2}+\frac{4}{3} \frac{|\theta|^{3} E\left(\left|Y_{n, t}\right|^{3}\right)}{\sigma^{3}(t)}\right)=\exp \left(-\theta^{2}\left(1-\frac{4}{3}|\theta| \frac{\Gamma_{3}(t)}{\sigma^{3}(t)}\right)\right)
$$

Pour conclure, si $|\theta| \leq \frac{1}{4 \frac{\Gamma_{3}(t)}{\sigma^{3}(t)}}$ alors $1-\frac{4}{3}|\theta| \frac{\Gamma_{3}(t)}{\sigma^{3}(t)} \geq 2 / 3$ et par conséquent $\left|\varphi_{Z_{t}}(\theta)\right|^{2} \leq e^{-2 \theta^{2} / 3}$.

Comme $\pi \sigma(t) \sim \sqrt{\frac{1}{6}} \frac{\pi^{2}}{r^{3 / 2}}$ lorsque $t$ tend vers 1 il suffit maintenant d'obtenir une majoration de la fonction caractéristique sur le domaine $\frac{1}{4 C_{3}} r^{-1 / 2} \leq|\theta| \leq \sqrt{\frac{1}{6}} \frac{\pi^{2}}{r^{3 / 2}}$.

Lemme 4.3 Soit $C$ une constante positive. Sous l'hypothèse $\frac{C}{r^{1 / 2}} \leq|\theta|<\pi \sigma(t)$ il existe un réel positif $B$ tel que l'on ait $\left|\varphi_{Z_{t}}(\theta)\right| \leq e^{-B / r}$.

\section{Démonstration.}

La méthode consiste à écrire

$\ln \left(\left|\varphi_{Z_{t}}(\theta)\right|\right)=\sum_{k \geq 1} \ln \left(\left|1+t^{k} e^{i k \theta / \sigma(t)}\right|\right)-\ln \left(1+t^{k}\right)$.

On développe

$$
\left|1+t^{k} e^{i k \theta / \sigma(t)}\right|^{2}=1+t^{2 k}+2 t^{k} \cos (k \theta / \sigma(t))
$$

et on écrit

$$
\begin{aligned}
\ln \left(\left|\varphi_{Z_{t}}(\theta)\right|\right) & =\frac{1}{2} \sum_{k \geq 1} \ln \left(1+t^{2 k}+2 t^{k} \cos (k \theta / \sigma(t))\right)-\ln \left(1+t^{2 k}+2 t^{k}\right) \\
& =\frac{1}{2} \sum_{k \geq 1} \ln \left(1+\frac{2 t^{k}(\cos (k \theta / \sigma(t))-1)}{1+t^{2 k}+2 t^{k}}\right) \\
& \leq \frac{1}{2} \sum_{k \geq 1} \frac{2 t^{k}(\cos (k \theta / \sigma(t))-1)}{1+t^{2 k}+2 t^{k}} \\
& \leq \frac{1}{4} \sum_{k \geq 1} t^{k}(\cos (k \theta / \sigma(t))-1)
\end{aligned}
$$

Or on a

$$
\sum_{k \geq 1} t^{k}\left(\cos (k \theta / \sigma(t))=\operatorname{Re}\left(\frac{t e^{i \theta / \sigma(t)}}{1-t e^{i \theta / \sigma(t)}}\right)=\frac{t \cos (\theta / \sigma(t))-t^{2}}{1-2 t \cos (\theta / \sigma(t))+t^{2}}\right.
$$


et puisque $C r \leq|\theta| / \sigma(t)<\pi$ alors $\cos (\theta / \sigma(t)) \leq \cos (C r)$. Par conséquent

$$
\sum_{k \geq 1} t^{k}\left(\cos (k \theta / \sigma(t)) \leq \frac{t \cos (C r)-t^{2}}{1-2 t \cos (C r)+t^{2}}\right.
$$

donc

$$
\ln \left(\left|\varphi_{Z_{t}}(\theta)\right|\right) \leq \frac{1}{4}\left(\frac{t \cos (C r)-t^{2}}{1-2 t \cos (C r)+t^{2}}-\frac{t}{1-t}\right) \backsim\left(\frac{1}{1+C^{2}}-1\right) r^{-1}
$$

On en déduit l'existence d'une constante $B>0$ telle que l'on ait :

$$
\left|\varphi_{Z_{t}}(\theta)\right| \leq e^{-B / r}
$$

Théorème 4.4 On a

$$
\lim _{t \rightarrow 1} \int_{-\pi \sigma(t)}^{\pi \sigma(t)}\left|\varphi_{Z_{t}}(\theta)-e^{-\theta^{2} / 2}\right| d \theta=0
$$

\section{Démonstration.}

D'après le lemme (4.3) :

$$
\left|\varphi_{Z_{t}}(\theta)\right| \leq e^{-B / r} \text { si } \frac{C}{r^{1 / 2}} \leq|\theta| \leq \pi \sigma(t), \text { avec } B>0
$$

et clairement

$$
e^{-\theta^{2} / 2} \leq e^{-C^{2} / 2 r}
$$

sous les mêmes conditions. On a donc, avec $D=\min \left(B, C^{2} / 2\right)$ :

$$
\begin{aligned}
\int_{\frac{C}{r^{1 / 2}} \leq|\theta| \leq \pi \sigma(t)}\left|\varphi_{Z_{t}}(\theta)-e^{-\theta^{2} / 2}\right| d \theta & \leq \int_{\frac{C}{r^{1 / 2}} \leq|\theta| \leq \pi \sigma(t)}\left|\varphi_{Z_{t}}(\theta)\right| d \theta+\int_{\frac{C}{r^{1 / 2}} \leq|\theta| \leq \pi \sigma(t)} e^{-\theta^{2} / 2} d \theta \\
& \leq e^{-D / r}\left(\pi \sigma(t)-\frac{C}{r^{1 / 2}}\right)
\end{aligned}
$$

et cette dernière quantité tend vers 0 lorsque $t$ tend vers 1 (i.e. lorsque $r$ tend vers 0 ).

Il reste à voir que

$$
\lim _{t \rightarrow 1} \int_{|\theta| \leq \frac{C}{r^{1 / 2}}}\left|\varphi_{Z_{t}}(\theta)-e^{-\theta^{2} / 2}\right| d \theta=0
$$

D'après le lemme (4.1), sur cet intervalle on a $\left|\varphi_{Z_{t}}(\theta)\right| \leq e^{-\theta^{2} / 3}$ donc

$$
\left|\varphi_{Z_{t}}(\theta)-e^{-\theta^{2} / 2}\right| \leq e^{-\theta^{2} / 3}+e^{-\theta^{2} / 2}
$$

et on peut conclure par le théorème de la convergence dominée.

\subsection{Application du théorème des équivalents}

On a choisi comme équivalent de la fonction $m$ lorsque $t$ tend vers 1 la fonction $m_{1}$ définie par

$$
m_{1}\left(e^{-r}\right)=\frac{\pi^{2}}{12} \frac{1}{r^{2}} .
$$

La définition de $\tau_{n}$ par l'égalité $m_{1}\left(\tau_{n}\right)=n$ se traduit, en posant $\tau_{n}=e^{-\rho_{n}}$, par

$$
m_{1}\left(e^{-\rho_{n}}\right)=n \quad \text { ce qui donne } \quad \rho_{n}=\frac{1}{2 \sqrt{3} \sqrt{n}} \pi
$$


et par conséquent

$$
\tau_{n}=e^{-\frac{1}{2 \sqrt{3} \sqrt{n}} \pi}
$$

On a aussi choisi comme équivalent de la fonction $\sigma$ lorsque $t$ tend vers 1 la fonction $\sigma_{1}$ définie par

ce qui donne

$$
\sigma_{1}\left(e^{-r}\right)=\sqrt{\frac{\pi^{2}}{6} \frac{1}{r^{3}}}
$$

$$
\sigma_{1}^{2}\left(\tau_{n}\right)=\sigma_{1}^{2}\left(e^{-\rho_{n}}\right)=\frac{\pi^{2}}{6} \frac{1}{\rho_{n}^{3}}=\frac{\pi^{2}}{6} \frac{1}{\left(\frac{1}{2 \sqrt{3} \sqrt{n}} \pi\right)^{3}}=\frac{4}{\pi}(n)^{3 / 2} \sqrt{3}
$$

La condition du théorème des équivalents :

$$
\frac{m\left(\tau_{n}\right)-m_{1}\left(\tau_{n}\right)}{\sigma_{1}\left(\tau_{n}\right)} \rightarrow 0 \text { quand } \tau_{n} \rightarrow 1
$$

est bien satisfaite car on a vu en 4.1.1. que $m\left(e^{-r}\right)=m_{1}\left(e^{-r}\right)+O\left(\frac{1}{r}\right)$, ce qui permet d'écrire

$$
\frac{m\left(\tau_{n}\right)-m_{1}\left(\tau_{n}\right)}{\sigma_{1}\left(\tau_{n}\right)}=\frac{m\left(e^{-\rho_{n}}\right)-m_{1}\left(e^{-\rho_{n}}\right)}{\sigma_{1}\left(e^{-\rho_{n}}\right)}=\frac{O\left(\frac{1}{\rho_{n}}\right)}{\sigma_{1}\left(e^{-\rho_{n}}\right)}=\frac{O\left(n^{\frac{1}{2}}\right)}{3^{\frac{1}{4}} \sqrt{\frac{4}{\pi}} n^{3 / 4}} \rightarrow 0
$$

Le théorème des équivalents (2.3) nous permet donc d'obtenir la formule asymptotique :

$$
a_{n} \sim \frac{f\left(\tau_{n}\right)}{\sqrt{2 \pi} \sigma_{1}\left(\tau_{n}\right) \tau_{n}^{n}}
$$

Il reste à donner un équivalent de

$$
f\left(\tau_{n}\right)=\prod_{k=1}^{+\infty}\left(1+e^{-\frac{1}{2 \sqrt{3} \sqrt{n}} \pi k}\right)
$$

Passons au logarithme

$$
\ln \left(f\left(\tau_{n}\right)\right)=\sum_{k \geq 1} \ln \left(1+e^{-\frac{1}{2 \sqrt{3} \sqrt{n}} \pi k}\right)
$$

Lemme 4.5 On a pour $\rho \rightarrow 0^{+}$

$$
\sum_{k=1}^{+\infty} \ln \left(1+e^{-\rho k}\right)=\frac{\pi^{2}}{12 \rho}-\frac{1}{2} \ln 2+O(\rho)
$$

\section{Démonstration.}

Appliquons la formule d'Euler-McLaurin :

$$
\sum_{k \geq 1} \ln \left(1+e^{-\rho k}\right)=\int_{1}^{+\infty} \ln \left(1+e^{-\rho x}\right) d x+\frac{1}{2} \ln \left(1+e^{-\rho}\right)-\rho \int_{1}^{+\infty} b_{1}(x) \frac{e^{-\rho x}}{1+e^{-\rho x}} d x
$$

a) Le terme $\int_{1}^{+\infty} \ln \left(1+e^{-\rho x}\right) d x$ :

On décompose l'intégrale : 


$$
\begin{aligned}
\int_{1}^{+\infty} \ln \left(1+e^{-\rho x}\right) d x & =\int_{0}^{\infty} \ln \left(1+e^{-\rho x}\right) d x-\int_{0}^{1} \ln \left(1+e^{-\rho x}\right) d x \\
& =\int_{0}^{+\infty} \sum_{n \geq 1} \frac{(-1)^{n-1}}{n} e^{-\rho n x} d x-\ln 2+O(\rho) \\
& =\sum_{n \geq 1} \frac{(-1)^{n-1}}{\rho n^{2}}-\ln 2+O(\rho)
\end{aligned}
$$

Donc

$$
\int_{1}^{+\infty} \ln \left(1+e^{-\rho x}\right) d x=-\ln 2+\frac{\pi^{2}}{12 \rho}+O(\rho)
$$

b) Le terme $\frac{1}{2} \ln \left(1+e^{-\rho}\right)$ :

$$
\frac{1}{2} \ln \left(1+e^{-\rho}\right)=\frac{1}{2} \ln 2+O(\rho)
$$

c) Le troisième terme :

On a

$$
-\rho \int_{1}^{+\infty} b_{1}(x) \frac{e^{-\rho x}}{1+e^{-\rho x}} d x=O\left(\rho e^{-\rho}\right)
$$

En effet la fonction $x \mapsto \frac{e^{-\rho x}}{1+e^{-\rho x}}$ est positive décroissante et elle tend vers 0 à l'infini. Comme la fonction $b_{1}$ est périodique, par le lemme d'Abel on obtient la majoration

$$
\left|\int_{1}^{+\infty} b_{1}(x) \frac{e^{-\rho x}}{1+e^{-\rho x}} d x\right| \leq C \frac{e^{-\rho}}{1+e^{-\rho}} \leq C e^{-\rho}
$$

\section{Conclusion}

D'après le lemme (4.5) avec $\rho=\rho_{n}$ on a

$$
\sum_{k \geq 1} \ln \left(1+e^{-\frac{1}{2 \sqrt{3} \sqrt{n}} \pi k}\right)=\frac{\pi \sqrt{n}}{2 \sqrt{3}}-\frac{1}{2} \ln 2+O\left(\frac{1}{\sqrt{n}}\right)
$$

donc

$$
f\left(\tau_{n}\right)=\prod_{k=1}^{+\infty}\left(1+e^{-\frac{1}{2 \sqrt{3} \sqrt{n}} \pi k}\right) \sim \frac{1}{\sqrt{2}} e^{\frac{\pi \sqrt{n}}{2 \sqrt{3}}}
$$

lorsque $n$ tend vers l'infini, ce qui donne la formule asymptotique des partitions restreintes:

$$
q(n) \sim \frac{1}{\sqrt{2}} e^{\frac{\pi \sqrt{n}}{2 \sqrt{3}}} \frac{1}{\sqrt{2 \pi} \sqrt{\frac{4}{\pi}(n)^{3 / 2} \sqrt{3}} e^{-\frac{\sqrt{n}}{2 \sqrt{3}} \pi}}=\frac{1}{4} \frac{e^{\frac{\pi \sqrt{n}}{\sqrt{3}}}}{3^{1 / 4} n^{3 / 4}}
$$




\section{Appendice}

\subsection{Démonstration du Lemme 3.3.}

Comme $\sup _{n \geq 1}\left|u_{n, t}\right| \rightarrow 0$ quand $t \rightarrow 1$, il existe $a<1$ tel que pour $\left.t \in\right] a, 1\left[\right.$ on a $\left|u_{n, t}\right|<1 / 2$ pour tout $n \geq 1$. Donc $\ln \left(1+u_{n, t}\right)$ est bien défini pour $\left.t \in\right] a, 1[$ et

$$
\ln \left(1+u_{n, t}\right)=\sum_{k=1}^{+\infty} \frac{(-1)^{k-1}}{k}\left(u_{n, t}\right)^{k}
$$

ce qui donne

$$
\left|\ln \left(1+u_{n, t}\right)-u_{n, t}\right| \leq\left|u_{n, t}\right|^{2} \sum_{k=2}^{+\infty} \frac{1}{k}\left|u_{n, t}\right|^{k-2} \leq\left|u_{n, t}\right|^{2} \sum_{k=2}^{+\infty}\left(\frac{1}{2}\right)^{k-2}=2\left|u_{n, t}\right|^{2}
$$

D'autre part la série $\sum_{n \geq 1}\left|u_{n, t}\right|$ est supposée convergente pour tout $\left.t \in\right] \alpha, 1\left[\right.$, donc la série $\sum_{n \geq 1}\left|u_{n, t}\right|^{2}$ est convergente si $t \in] \sup (a, \alpha), 1\left[\right.$. On en déduit que la série $\sum_{n \geq 1} \ln \left(1+u_{n, t}\right)$ est convergente si $t \in] \sup (a, \alpha), 1\left[\right.$ et il en est donc de même du produit infini $\prod_{n \geq 1}\left(1+u_{n, t}\right)$.

D'autre part, pour tout $N \geq 1$ on a

$$
\left|\sum_{n=1}^{N} \ln \left(1+u_{n, t}\right)-\sum_{n=1}^{N} u_{n, t}\right| \leq \sum_{n=1}^{N}\left|\ln \left(1+u_{n, t}\right)-u_{n, t}\right| \leq 2 \sum_{n=1}^{N}\left|u_{n, t}\right|^{2}
$$

Comme

$$
\sum_{n=1}^{N}\left|u_{n, t}\right|^{2} \leq \sup _{n \geq 1}\left|u_{n, t}\right| \sum_{n=1}^{N}\left|u_{n, t}\right| \leq M \sup _{n \geq 1}\left|u_{n, t}\right|
$$

on en déduit que $\sum_{n=1}^{+\infty}\left|u_{n, t}\right|^{2} \leq M \sup _{n \geq 1}\left|u_{n, t}\right|$ et que

$$
\lim _{N \rightarrow+\infty}\left|\sum_{n=1}^{N} \ln \left(1+u_{n, t}\right)-\sum_{n=1}^{N} u_{n, t}\right| \leq 2 M \sup _{n \geq 1}\left|u_{n, t}\right|
$$

Donc

$$
\left|\sum_{n=1}^{+\infty} \ln \left(1+u_{n, t}\right)-\sum_{n=1}^{+\infty} u_{n, t}\right| \leq 2 M \sup _{n \geq 1}\left|u_{n, t}\right| .
$$

Pour conclure il suffit de prendre la limite quand $t \rightarrow 1$, on obtient

$$
\lim _{t \rightarrow 1} \sum_{n=1}^{+\infty} \ln \left(1+u_{n, t}\right)=\lim _{t \rightarrow 1} \sum_{n=1}^{+\infty} u_{n, t}=S
$$

En passant à l'exponentielle on obtient

$$
\lim _{t \rightarrow 1} \prod_{n=1}^{+\infty}\left(1+u_{n, t}\right)=e^{S}
$$




\subsection{Démonstration du Lemme de Cramér (Lemme 4.2)}

Démonstration. (voir Cramér [3], Chung [2] p. 210)

Soit $Y$ une variable aléatoire indépendante de $Z$ et de même loi. On a

$$
\left|\varphi_{Z}(\xi)\right|^{2}=\varphi_{Z}(\xi) \overline{\varphi_{Y}(\xi)}=E\left(e^{i \xi Z}\right) \overline{E\left(e^{i \xi Y}\right)}=E\left(e^{i \xi(Z-Y)}\right)
$$

ce qui permet d'écrire

$$
\left|\varphi_{Z}(\xi)\right|^{2}=\int_{\mathbb{R}^{2}} e^{i \xi(z-y)} d P_{Z}(z) d P_{Y}(y)=\int_{\mathbb{R}^{2}} \cos (\xi(z-y)) d P_{Z}(z) d P_{Y}(y)
$$

En utilisant la majoration

$$
\cos (u) \leq 1-\frac{u^{2}}{2}+\frac{|u|^{3}}{6}
$$

que l'on peut obtenir à l'aide de la formule de Taylor d'ordre deux avec reste intégral, on en déduit que

$$
\left|\varphi_{Z}(\xi)\right|^{2} \leq 1-\frac{\xi^{2}}{2} \int_{\mathbb{R}^{2}}(z-y)^{2} d P_{Z}(z) d P_{Y}(y)+\frac{|\xi|^{3}}{6} \int_{\mathbb{R}^{2}}|z-y|^{3} d P_{Z}(z) d P_{Y}(y)
$$

La première intégrale n'est autre que $2 E\left(Z^{2}\right)$. Pour la deuxième on utilise la majoration

$$
|z-y|^{3} \leq 4|z|^{3}+4|y|^{3}
$$

ce qui permet de majorer l'intégrale par $8 E\left(|Z|^{3}\right)$.

On obtient finalement

$$
\left|\varphi_{Z}(\xi)\right|^{2} \leq 1-\xi^{2} E\left(Z^{2}\right)+\frac{4}{3}|\xi|^{3} E\left(|Z|^{3}\right) \leq e^{-\xi^{2} E\left(Z^{2}\right)+\frac{4}{3}|\xi|^{3} E\left(|Z|^{3}\right)}
$$

Pour la seconde partie du lemme, si $|\xi| \leq \frac{1}{2} \frac{E\left(X^{2}\right)}{E\left(|X|^{3}\right)}$ il suffit de remarquer que

$$
-\xi^{2} E\left(Z^{2}\right)+\frac{4}{3}|\xi|^{3} E\left(|Z|^{3}\right)=-\xi^{2}\left[E\left(Z^{2}\right)-\frac{4}{3}|\xi| E\left(|Z|^{3}\right)\right] \leq-\xi^{2} \frac{1}{3} E\left(Z^{2}\right)
$$

\section{Remerciements}

Nous remercions Mesdames Cécile Fouilhé et Noëmie El Qotbi pour l'intérêt qu'elles ont porté à l'étude de l'article de Luis Báez-Duarte.

\section{Références}

[1] Báez-Duarte, L., "Hardy-Ramanujan's Asymptotic Formula for Partitions and the Central Limit Theorem," Advances in Mathematics 125, 114-120 (1997).

[2] Chung, K.L., "A Course in Probability Theory, 3rd ed." Academic Press, San Diego [CA] (2001).

[3] Cramér, H., "Random Variables and Probability Distributions," 2nd ed., Cambridge Univ. Press, Cambridge (1963).

[4] Erdös, P., Lehner, J., "The Distribution of the Number of Summands in the Partitions of a Positive Integer," Duke Mathematical Journal Vol. 8, No.2 (June, 1941)

[5] Hayman, W.K., “A Generalisation of Stirling's Formula," J. Reine Angew. Mat. 196, Nos. 1/2, 67-95 (1956).

[6] Ingham, A.E., "A Tauberian Theorem for Partitions," The Annals of Mathematics, Second Series, Vol. 42, No. 5, 1075-1090 (Dec., 1941)

[7] Rosenbloom, P.C., "Probability and Entire Functions," Studies in Mathematical Analysis and Related Topics, Vol. 45, 325-332, Stanford Univ. Press, Palo Alto, CA (1962). 